\title{
Clearing of corneal argyrosis by YAG laser
}

\author{
O GEYER, L ROTHKOFF, AND M LAZAR
}

From the Department of Ophthalmology, Ichilov Hospital, Tel Aviv, Israel

SUMMARY A 75-year-old woman with corneal argyrosis was treated by a Q-switched Nd-YAG laser iridotomy after acute angle closure glaucoma. Each laser shot caused clearance of the argyrotic deposits anterior to the iridotomy site. The same effect was seen when a preventive iridotomy was performed in the other eye. The areas of clear cornea remained unchanged for a follow-up period of eight months.

Corneal argyrosis is the deposition of silver granules in the deep stroma and Descemet's membrane following the use of topical silver nitrate.' The cornea takes on a grey appearance by reflected light. The deposition is considered to be permanent, and no efficient method of removal has been reported.

\section{Case report}

A 75-year-old woman was admitted to our department because of an acute attack of angle closure glaucoma in her right eye. On examination we found the typical bluish grey pigmentation of the conjunctiva, particularly in the lower fornix, and the grey colour of the deep cornea which are characteristic of argyrosis. The patient recalled having received extensive silver nitrate therapy for trachoma 50 years previously.

After the acute attack had been arrested medically a laser iridotomy was performed with a Q-switched Nd-YAG laser (Lasag). A single burst of $10 \mathrm{~mJ}$ through an iris contact lens was used with the heliumneon beam focused carefully on the iris. Multiple shots were necessary to achieve an iridotomy. To our surprise each laser shot caused a bubble in the deep

Correspondence to O Geyer, MD, Department of Ophthalmology, Ichilov Hospital, 6 Weizman Street. Tel Aviv 64239. Israel. cornea, with disappearance of the argyrotic deposit immediately anterior to the iridotomy site. Each window of clear cornea had an oval shape and was slightly larger in area than the iridotomy (Fig. 1). The same effect was seen when a preventive iridotomy was performed in the patient's left eye. The areas of clear cornea remained unchanged for the follow-up period of eight months.

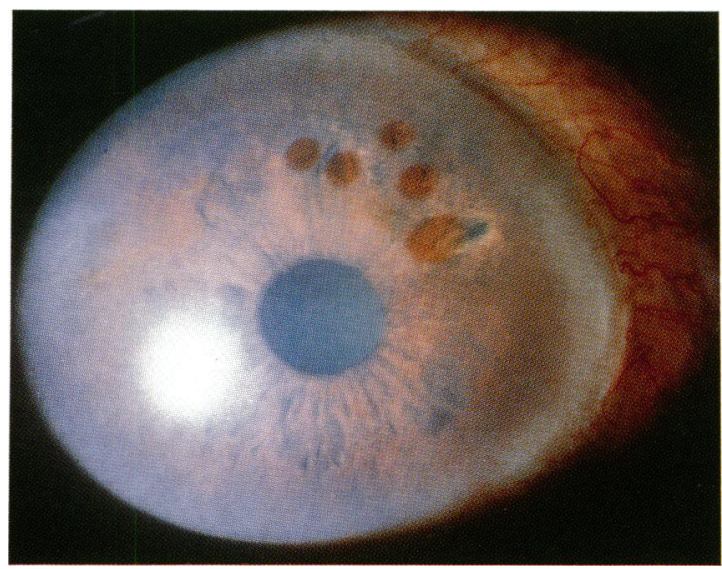

Fig. 1 Clear areas in argyrotic cornea after YAG laser treatment. 


\section{Discussion}

To the best of our knowledge the clearing of silver deposits in the cornea with YAG laser has not been previously reported. It is most probably due to partial absorption of the laser beam by the silver deposits causing their optical breakdown and vapourisation. This was evidenced by bubble formation in the deep cornea with each laser shot.

Because corneal argyrosis is a rare finding today we do not expect the YAG laser to have any clinical use in its treatment. However, perhaps this finding can serve as a basis for using YAG laser in attempts to clear other precipitations and pigmentations in the cornea, such as blood staining, which are much more common.

\section{Reference}

1 Duke-Elder WS. System of ophthalmology. London: Kimpton. 1972; 8: 990.

Accepted for publication 30 June 1989. 